\title{
Pyogenic Granuloma in Pregnancy: A Case Report
}

\author{
Fatma Ucan Yarkac ${ }^{* 1}$ and Ozge Gokturk ${ }^{2}$ \\ ${ }^{1}$ Department of Periodontology, Gaziosmanpasa University, Faculty of Dentistry, Turkey
}

${ }^{2}$ Department of Periodontology, Abant Izzet Baysal University, Faculty of Dentistry, Turkey

Received: May 22, 2018; Published: May 31, 2018

*Corresponding author: Fatma Ucan Yarkac, Department of Periodontology, Gaziosmanpasa University, Faculty of Dentistry, Tokat 60000, Turkey

\begin{abstract}
Pyogenic granuloma is thought to be an extreme tissue response to trauma or local irritation. Hormonal changes also trigger the formation of the lesion. Clinically, this lesion is usually found as single nodules or vesicular papules with smooth or lobular surfaces. They can be seen from a few millimeters to a few centimeters at any size. It is known that pyogenic granuloma occurs more frequently in the teeth $(75 \%$ of all cases). In this article, we presented a laser-assisted surgical treatment of a gingival pyogenic granuloma consisting of the right molar region of the mandible in a 22-year-old pregnant woman.
\end{abstract}

Keywords: Pyogenic granuloma; Laser; Pregnancy; Surgery

\section{Background}

Pyogenic granuloma (PG), a nonneoplastic lesion of the oral cavity, is thought to occur as a reactive tissue-reaction to local irritation or trauma [1]. Clinically, PG generally occurs as a solitary exophytic growth that can be sessile or pedunculated with a smooth or lobulated surface. The color of the growth is red in younger lesions and with maturity; it becomes more pink and fibrous as the vascularity decreases. It may be painful, especially if located in an uncomfortable area of the body. PG can grow quickly and will frequently bleed profusely with little or no trauma. The most frequent intraoral site is the gingiva, maxilla being more involved than mandible. It sometimes consists of extra gingivally on the lips, tongue, buccal mucosa, and palate [2]. PG mostly occurs in young females in the second decade of life. Because of a vascular impact owing to hormonal changes. It is generally thought that that female sex hormones play significant roles in its pathogenesis [1,3].

Many treatment methods have been developed for PG. Before treatment, the etiology must be identified and eradicated. When the lesion is small, painless and there is no bleeding, oral prophylaxis, removal of causative irritants (foreign materials, the source of trauma), and follow-up are recommended [4]. If lesions are large, they are treated by a whole oral prophylaxis followed by surgical excision using gingivectomy or flap surgery methods with additional therapy $[2,5]$. Lesions removed during pregnancy can have a higher recurrence ratio [2]. Recurrence occurs after incomplete excision, failure to remove etiologic factors or re-injury of the area.
The elimination of this lesion can be carried out using different approaches. For removing PG, cryosurgery, scalpel, and laser might be used $[2,5,6]$. Laser surgery is the preferred method of good patient comfort, easy to application and short operation time without bleeding and suturing [7].

Diode laser, CO2 (Carbon Dioxide Laser), Nd: YAG (Neodymium-Doped Yttrium, Aluminium Garnet) and Er: YAG (Erbium-Doped Yttrium Aluminum Garnet) have reportedly been successful in treating mucosal pyogenic granulomas $[5,7,8]$. The possibility of recurrence of the lesion seems that the use of laser will reduce this recurrence rate [7]. The goal of this article is to report a case of gingival pyogenic granuloma occurring on the right buccal mucosa in a female and to distinguish this lesion from other similar lesions of the buccal mucosa, with special emphasis on the diagnosis and treatment of this condition.

\section{Case Description}

A 22-year-old female patient noticed with the principal complaint of growth on the right mandibular molar area with 3-month duration in the second trimester of pregnancy. History of presenting illness showed that the growth was gradual in onset, initially small in dimension now increased to attain the present size. There was also a history of occasional bleeding from the growth during chewing. The patient's medical history and family were not existing of PG. Extraoral examination demonstrated no swelling or facial asymmetry. Intraoral examination showed a solitary exophytic, pe- 
dunculated growth on the right side of buccal mucosa at the level of the occlusal plane. The exophytic growth seems erythematous with grayish white borders with lobulated surface. The growth measured of about $15 \times 10 \times 5 \mathrm{~mm}$ in diameter, which was soft to firm in consistency and bled on provocation (Figure 1). There was no evidence of pus discharge from the lesion. Based on the history and clinical appearance of the lesion, provisional diagnosis of benign exophytic growth of right buccal mucosa was considered. And the differential diagnosis included traumatic fibroma, pyogenic granuloma, and capillary hemangioma. The lesion, which was retained with the help of a hemostat, was excised by using a lancet and removed until debris and soft tissue was eliminated. Coagulation was performed in the surgical area by diode laser (hemostasis mode, in continuous mode, power output $0.5 \mathrm{~W}$, frequency $50 \mathrm{~Hz}$ ). It took five minutes to complete the procedure. Then, the lesion sent for histopathological examination. Figure 2 shows the macroscopic appearance of the excised specimen. Histopathologically, hematoxylin-eosin (H\&E) stained section confirmed the clinical diagnosis of PG (Figure 3). The operation area has improved and there was no recurrence in the patient after post-operative 6 months (Figure 4).

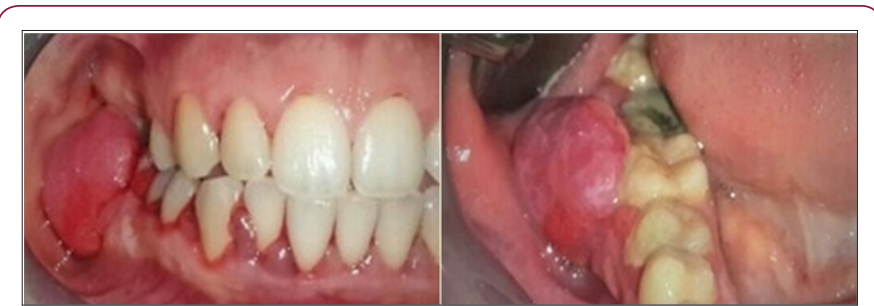

Figure 1: Pre-operative image showing pyogenic granuloma.

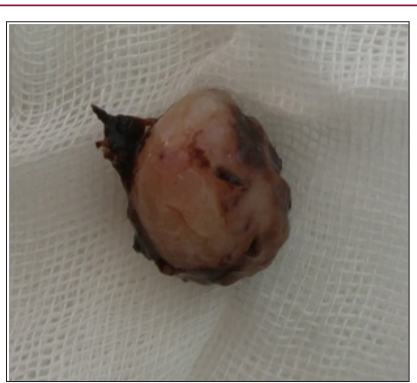

Figure 2: The macroscopic appearance of the excised lesion.

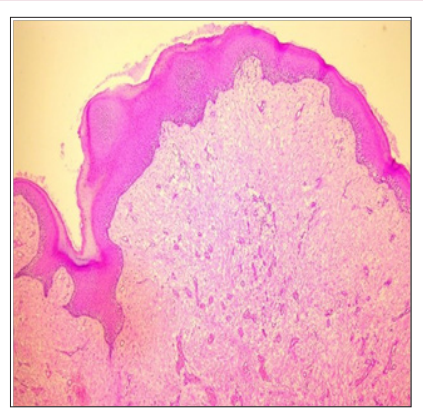

Figure 3: Histological appearance of a pyogenic granuloma.

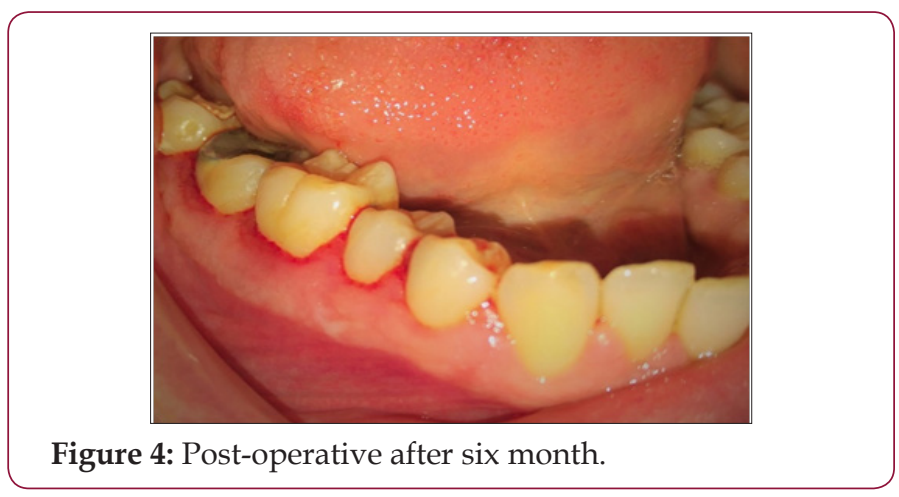

\section{Discussion}

Pyogenic granuloma is a reactive inflammatory course occupied with proliferating vascular channels, immature fibroblastic connective tissue, and scattered inflammatory cells [1]. Because it is a reactive tumor-like lesion, chronic low-grade irritation, traumatic injury, hormonal factors and certain kind of drugs could be thought as etiologic agents [2]. PG of the gingiva occurs in up to $5 \%$ of pregnancies [5]. Therefore the terms "pregnancy tumor" and "granuloma gravidarum" are frequently utilized [9]. The hormonal instability coincident with pregnancy heightens the organism's reply to irritation but, bacterial plaque and gingival inflammation are essential for PG [1-3]. Treatment of PG requires a whole surgical excision [4]. Recurrence of PG after excision is a known complication but can be prevented. Therefore, additional treatments are needed to increase the effectiveness of surgical treatment and to prevent recurrence. The hemostatic feature of the laser is very significant in excising exophytic lesion $[7,10]$. It can be used to stopping bleeding in the field by its contract vascular wall collagen [8]. This gives good visibility and precision in the operation area. The tissue coagulation by diode laser is a clinical importance. The laser has no need for suturing, no bleeding at the site of surgery, the surgical period was markedly minimized and patients were protected from potential high-risk infection $[7,10]$. In some studies, there was a problem of coagulation in some PGs then those specific areas were coagulated with Nd: YAG or Diode lasers and thus they were successfully treated $[11,12]$. Kocaman et al. [11] used Nd: YAG laser in order to remove PG and deduced that the use of Nd: YAG laser in the treatment of pyogenic granuloma reduced bleeding during surgery, with a consequent promoted rapid postoperative hemostasis, and has better patient comfort [11]. The use of a $940 \mathrm{~nm}$ diode laser was an accomplished therapy option for decreasing the risk of post-operative infection and impaired healing, increasing of hemostasis as well as increasing the patient's comfort $[13,14]$. The laser treatment can provide hemostasis for very large and vascularized lesions.

\section{Conclusion}

Pyogenic granuloma is generally encountered soft tissue enlargements. The laser-assisted surgical treatment of the lesion can be considered as a good modality for very large and vascularized lesions. Laser treatment can prevent recurrences of PG. 


\section{Acknowledgment}

I would like to express my very great appreciation to Prof. Dr. Resit Dogan Koseoglu for his histologically evaluated the excisional biopsy material at this research.

\section{References}

1. Ramirez K, Bruce G, Carpenter W (2002) Pyogenic granuloma: case report in a 9-year-old girl. Gen Dent 50(3): 280-281.

2. Jafarzadeh H, Sanatkhani M, Mohtasham N (2006) Oral pyogenic granuloma: a review. J Oral Sci 48(4): 167-175.

3. Patil K, Mahima V, Lahari K (2006) Extragingival pyogenic granuloma. Indian J Dent Res 17(4): 199.

4. Sills ES, Zegarelli DJ, Hoschander MM, Strider WE (1996) Clinical diagnosis and management of hormonally responsive oral pregnancy tumor (pyogenic granuloma). J Reprod Med 41(7): 467-470.

5. White J, Chaudhry SI, Kudler JJ, Sekandari N, Schoelch ML, Silverman S Jr (1998) Nd: YAG and CO2 laser therapy of oral mucosal lesions. J Clin Laser Med Surg 16(6): 299-304.

6. Ishida M, Ramos-e-Silva M (1998) Cryosurgery in oral lesions. Int J Dermatol 37(4): 283-285.

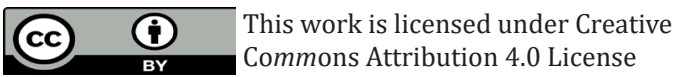

Submission Link: https://biomedres.us/submit-manuscript.php
7. Rai S, Kaur M, Bhatnagar P (2011) Laser: a powerful tool for treatment of pyogenic granuloma. J Cutan Aesthet Surg 4(2): 144.

8. Fekrazad R, Chiniforush N (2014) One visit providing desirable smile by laser application. J Lasers Med Sci 5(1): 47-50.

9. Mubeen K, Vijayalakshmi K, Abhishek R (2011) Oral pyogenic granuloma with mandible involvement: An unusual presentation. J Dent Oral Hyg 3(1): 6-9.

10. Kirschner RE, Low DW (1999) Treatment of pyogenic granuloma by shave excision and laser photocoagulation. Plast Reconstr Surg 104(5): 1346-1349.

11. Kocaman G, Belduz N, Erdogan C, Ozbek E, Sadik E, Kara C (2014) The use of surgical Nd: YAG laser in an oral pyogenic granuloma: A case report. J Cosmet Laser Ther 16(4): 197-200.

12. Akbulut N, Kursun ES, Tumer MK, Kamburoglu K, Gulsen U (2013) Is the 810-nm diode laser the best choice in oral soft tissue therapy? Eur J Dent 7(2): 207-211.

13. Iyer VH, Sasikumar R (2013) Management of oral pyogenic granuloma with $940 \mathrm{~nm}$ diode laser: a rare case report. Int J Laser Dent 3(3): 100.

14. Al-Mohaya MA, Al-Malik AM (2016) Excision of oral pyogenic granuloma in a diabetic patient with 940nm diode laser. Saudi Med J 37(12): 13951400 .

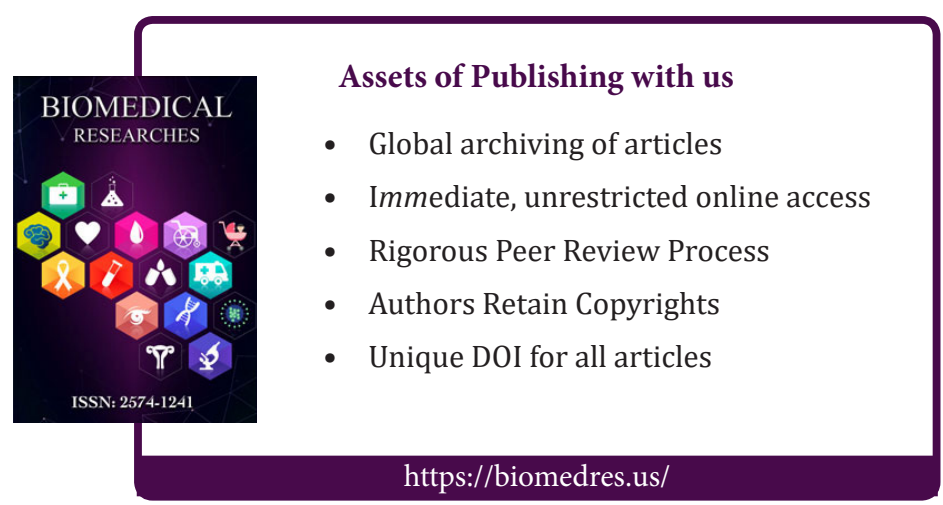

